\title{
THE NUMERICAL SOLUTION \\ OF FIRST-KIND LOGARITHMIC-KERNEL INTEGRAL EQUATIONS ON SMOOTH OPEN ARCS
}

\author{
KENDALL E. ATKINSON AND IAN H. SLOAN
}

ABSTRACT. Consider solving the Dirichlet problem

$$
\begin{aligned}
& \Delta u(P)=0, \quad P \in \mathbb{R}^{2} \backslash S, \\
& u(P)=h(P), \quad P \in S, \\
& \sup _{P \in \mathbb{R}^{2}}|u(P)|<\infty,
\end{aligned}
$$

with $S$ a smooth open curve in the plane. We use single-layer potentials to construct a solution $u(P)$. This leads to the solution of equations of the form

$$
\int_{S} g(Q) \log |P-Q| d S(Q)=h(P), \quad P \in S .
$$

This equation is reformulated using a special change of variable, leading to a new first-kind equation with a smooth solution function. This new equation is split into a principal part, which is explicitly invertible, and a compact perturbation. Then a discrete Galerkin method that takes special advantage of the splitting of the integral equation is used to solve the equation numerically. A complete convergence analysis is given; numerical examples conclude the paper.

\section{INTRODUCTION}

We consider the numerical solution of

$$
\int_{S} g(Q) \log |P-Q| d S(Q)=h(P), \quad P \in S,
$$

with $S$ a smooth open contour in the plane. This equation arises in a variety of contexts, one being the study of elasticity crack problems in the plane. We propose a new numerical method for solving (1.1), and then use it to solve some potential theory problems in the plane. The method takes account of the expected singularities in $g$ at the ends of the contour in an entirely natural way, and the method is shown to converge rapidly when the curve $S$ and the data are sufficiently smooth. We limit the functions $g$ and $h$ to be real, although the following development extends easily to the complex case.

Let $S$ have a parametrization

$$
r(x)=(\xi(x), \eta(x)), \quad-1 \leq x \leq 1,
$$

Received November 4, 1988; revised December 18, 1989.

1980 Mathematics Subject Classification (1985 Revision). Primary 65R20; Secondary 31A10, $35 \mathrm{C} 05,65 \mathrm{~N} 99$. 
with $\left|r^{\prime}(x)\right| \neq 0,-1 \leq x \leq 1$. To simplify the analysis, assume $r(x)$ is $C^{\infty}$. Following Yan and Sloan [12], we make the additional change of variable

$$
t=\cos ^{-1}(x), \quad-1 \leq x \leq 1 .
$$

The equation (1.1) can now be written as

$$
-\frac{1}{\pi} \int_{0}^{\pi} \rho(\tau) \log |a(t)-a(\tau)| d \tau=f(t), \quad 0 \leq t \leq \pi,
$$

with

$$
\begin{aligned}
a(t) & =r(\cos t), \\
\rho(t) & =g(a(t))\left|r^{\prime}(\cos t)\right| \sin t, \\
f(t) & =-\frac{1}{\pi} h(a(t)) .
\end{aligned}
$$

Note that $a \in C^{\infty}(\mathbb{R})$.

An advantage of the formulation (1.4), (1.5) is that the singularities in $g$ in the original problem now become explicit: for if the solution $\rho$ of (1.4) is smooth, then $g$ automatically has the expected $x^{-1 / 2}$ type singularities at the two ends of the contour, because of the factor $\sin t$ in (1.5). A smooth solution $\rho$ of (1.4) arises naturally if $h$ in (1.1) is smooth. This follows from the observation that the functions $a$ and $f$ above are even, $2 \pi$-periodic functions; and if $h \in C^{\infty}(S)$, then $f \in C^{\infty}(\mathbb{R})$. If we define $\rho$ to be an even function, then the integral in (1.4) can be replaced by one half of the integral over a full period. Since the resulting equation can now be viewed as a problem involving smooth functions on the circle, we must expect that $\rho$ can be considered a $2 \pi$-periodic $C^{\infty}$ function on the whole line, and we shall see below that this is indeed the case.

The equation (1.4) is split as

$$
\begin{gathered}
\left(A_{e}+B_{e}\right) \rho=f, \\
A_{e} \rho(t)=-\frac{1}{\pi} \int_{0}^{\pi} \rho(\tau) \log \left[2 e^{-1}|\cos t-\cos \tau|\right] d \tau, \\
B_{e} \rho(t)=\int_{0}^{\pi} \rho(\tau) b_{e}(t, \tau) d \tau, \\
b_{e}(t, \tau)= \begin{cases}-\frac{1}{\pi} \log \left|\frac{e}{2} \frac{a(t)-a(\tau)}{\cos t-\cos \tau}\right|, & t-\tau, t+\tau \neq 2 \pi m, \\
-\frac{1}{\pi} \log \left|\frac{e}{2} r^{\prime}(\cos t)\right|, & t-\tau \text { or } t+\tau=2 \pi m,\end{cases}
\end{gathered}
$$

with $m$ an arbitrary integer. With the assumptions on $r(x)$, it can be shown that $b_{e}(t, \tau)$ is a $C^{\infty}$ function of $t$ and $\tau$. It is also $2 \pi$-periodic and even, with respect to each variable.

The equation $A_{e} \rho=f$ is invertible explicitly if the Fourier cosine series of $f$ on $[0, \pi]$ is known. This will be used in $\S 3$ to define a Galerkin method with trigonometric trial functions for solving (1.6), and a corresponding discrete 
Galerkin method is defined and analyzed in $\S \S 4$ and 5. In $\S \S 6$ and 7 we discuss the application of these results to the solution of Laplace's equation, and we give some numerical examples. Section 2 contains preliminaries for putting (1.6) into an appropriate function space setting. The methods and results of this paper follow closely those of Atkinson [3], which defined and analyzed a discrete Galerkin method for solving (1.1) when $S$ is a simple closed curve.

A solvability analysis is given in Yan and Sloan [12], which we summarize here. Assume the transfinite diameter $C_{S} \neq 1$ for the curve $S$, and assume $S$ is $C^{\infty}$. Further, assume the function $f$ is differentiable, with $f^{\prime} \in L^{2}(0, \pi)$. Then the equation (1.6) has a unique solution $\rho \in L^{2}(0, \pi)$. This result generalizes to Sobolev spaces of even periodic functions, which are defined in the following section. The concept of transfinite diameter is described in Yan and Sloan [12]. For potential theory problems with $C_{S}=1$, the problem can be rescaled to give a new integral equation with $C_{S} \neq 1$. A further discussion of solving potential theory problems using $(1.1)$ is given in $\S 6$.

For other results on the numerical solution of (1.1) with $S$ an open curve, see Gladwell and Coen [7], Costabel, Ervin, and Stephan [6], and Sloan and Spence [11]. The paper of Gladwell and Coen contains a method related to one developed here, although no analysis is given. For the integral equation (1.1) with $S$ a closed curve, a numerical method similar to that of Atkinson [3] is presented and analyzed in McLean [10, Chapter 5]. The results of McLean can be generalized to (1.1) with $S$ an open curve, including an analysis of the effect of numerical integration in the implementation of the Galerkin method presented below in $\S 3$. Related boundary integral equations for the planar linear elasticity equations in a region exterior to an arc are given in Hsiao, Stephan, and Wendland [8], although their numerical methods are very different than those presented here.

\section{Preliminaries}

For our discussion of the operator $A_{e}$, we quote freely from Yan and Sloan [12]. For $t \geq 0$, let $H^{t}$ denote the Sobolev space of functions

$$
\begin{gathered}
\rho(s)=\frac{1}{\sqrt{2 \pi}} \sum_{m=-\infty}^{\infty} \hat{\rho}(m) e^{i m s}, \\
\hat{\rho}(m)=\frac{1}{\sqrt{2 \pi}} \int_{0}^{2 \pi} \rho(s) e^{-i m s} d s
\end{gathered}
$$

whose Fourier coefficients $\hat{\rho}(m)$ satisfy

$$
\|\rho\|_{t} \equiv\left[|\hat{\rho}(0)|^{2}+\sum_{|m|>0}|m|^{2 t}|\hat{\rho}(m)|^{2}\right]^{1 / 2}<\infty .
$$

It is well known that if $t>\frac{1}{2}$, then $H^{t} \subset C_{p}(2 \pi)$, the space of $2 \pi$-periodic 
continuous functions. More precisely, for $t>\frac{1}{2}$,

$$
\|\rho\|_{\infty} \leq\left[\frac{1+2 \zeta(2 t)}{\pi}\right]^{1 / 2}\|\rho\|_{t}
$$

with $\|\rho\|_{\infty}$ denoting the uniform norm of $\rho$.

We will be especially interested in the subspace $H_{e}^{t}$ of even periodic functions,

$$
H_{e}^{t}=\left\{f \in H^{t} \mid f(-s) \equiv f(s)\right\} .
$$

More precisely, $H_{e}^{t}$ is the closure of the space of even periodic $C^{\infty}$ functions under the norm $\|\cdot\|_{t}$. Then (2.1)-(2.3) become, respectively, for $\rho \in H_{e}^{t}$ :

$$
\begin{gathered}
\rho(s)=\sqrt{\frac{2}{\pi}}\left[\frac{1}{2} \hat{\rho}(0)+\sum_{m=1}^{\infty} \hat{\rho}(m) \cos (m s)\right], \\
\hat{\rho}(m)=\sqrt{\frac{2}{\pi}} \int_{0}^{\pi} \rho(s) \cos (m s) d s, \quad m \geq 0, \\
\|\rho\|_{t}=\left[|\hat{\rho}(0)|^{2}+2 \sum_{m=1}^{\infty} m^{2 t}|\hat{\rho}(m)|^{2}\right]^{1 / 2} .
\end{gathered}
$$

These are based on specializing (2.1)-(2.3), using the assumption that $\rho$ is even. Although (2.2) and (2.5) are different formulas, they give the same result when $\rho \in H_{e}^{t}$.

We will regard the operator $A_{e}$ of $(1.7)$ as an operator on $H_{e}^{t}$. For any $\rho \in H_{e}^{t}$, it can be shown that

$$
A_{e} \rho(s)=\sqrt{\frac{2}{\pi}}\left[\frac{1}{2} \hat{\rho}(0)+\sum_{m=1}^{\infty} \frac{\hat{\rho}(m)}{m} \cos (m s)\right] .
$$

Then

$$
A_{e}: H_{e}^{t} \underset{\text { onto }}{\stackrel{1-1}{\longrightarrow}} H_{e}^{t+1}, \quad t \geq 0
$$

with

$$
\left\|A_{e} \rho\right\|_{t+1}=\|\rho\|_{t} \text {. }
$$

The operator $A_{e}$ is invertible on $H^{t}, t \geq 1$, with

$$
A_{e}^{-1} \rho(s)=\sqrt{\frac{2}{\pi}}\left[\frac{1}{2} \hat{\rho}(0)+\sum_{m=1}^{\infty} m \hat{\rho}(m) \cos (m s)\right] \text {. }
$$

It will be useful to introduce a related operator $A$ which maps from $H^{t}$ to $H^{t+1}$ :

$$
A \rho(s)=\frac{1}{\sqrt{2 \pi}}\left[\hat{\rho}(0)+\sum_{|m|>0} \frac{\hat{\rho}(m)}{|m|} e^{i m s}\right],
$$


where $\rho \in H^{t}$ is given in (2.1). This is a representation of the integral operator

$$
A \rho(s)=-\frac{1}{\pi} \int_{0}^{2 \pi} \rho(\sigma) \log \left|2 e^{-1 / 2} \sin \left(\frac{s-\sigma}{2}\right)\right| d \sigma
$$

which is closely related to (1.1) for the case in which $S$ is the unit circle. From (2.11), it easily follows that

$$
A: H^{t} \underset{\text { onto }}{\stackrel{1-1}{\longrightarrow}} H^{t+1}
$$

and

$$
\|A \rho\|_{t+1}=\|\rho\|_{t} .
$$

A Fourier series representation of $A^{-1}$ follows easily from (2.11).

The main use of $A$ in this paper lies in the fact that

$$
A_{e} \rho=A \rho, \quad \rho \in H_{e}^{t} .
$$

Thus, results on $A$ can be used in investigating $A_{e}$. The integral equation (1.1) with $S$ a smooth simple closed curve was investigated in Atkinson [3]. Some of those results will be used here by means of (2.13).

\section{GALERKIN'S METHOD}

Let $\mathscr{X}_{n}$ denote the even trigonometric polynomials of degree $\leq n$. The standard basis for $\mathscr{X}_{n}$ is

$$
\left\{\varphi_{j}(s)=\cos (j s) \mid 0 \leq j \leq n\right\} .
$$

Restricting attention to the interval $[0, \pi]$, let $P_{n}$ denote the orthogonal projection of $H_{e}^{0} \equiv L^{2}(0, \pi)$ onto $\mathscr{X}_{n}$ :

$$
P_{n} \rho(s)=\sqrt{\frac{2}{\pi}}\left[\frac{1}{2} \hat{\rho}(0)+\sum_{m=1}^{n} \hat{\rho}(m) \cos (m s)\right],
$$

with $\rho$ given in (2.4). Note that $P_{n} \rho$ is simply the partial sum of the Fourier cosine series for $\rho$.

The Galerkin method for solving (1.6) consists of solving

$$
P_{n}\left(A_{e}+B_{e}\right) \rho_{n}=P_{n} f, \quad \rho_{n} \in \mathscr{X}_{n} .
$$

It is easily shown that

$$
P_{n} A_{e}=A_{e} P_{n}
$$

Thus, (3.3) can be rewritten as

$$
\left(A_{e}+P_{n} B_{e}\right) \rho_{n}=P_{n} f, \quad \rho_{n} \in \mathscr{X} \equiv L^{2}(0, \pi),
$$

with the solution $\rho_{n}$ automatically belonging to $\mathscr{X}_{n}$.

To analyze the convergence of $(3.5)$ in $L^{2}(0, \pi)$, consider instead the equivalent equation

$$
\left(I+P_{n} A_{e}^{-1} B_{e}\right) \rho_{n}=P_{n} A_{e}^{-1} f,
$$


using $A_{e}^{-1} P_{n}=P_{n} A_{e}^{-1}$. From the smoothness of $b_{e}(s, \sigma)$ in (1.9), the operator $B_{e}$ is compact from $H_{e}^{0}$ into $H_{e}^{t}$, for all $t \geq 0$. Thus $A_{e}^{-1} B_{e}$ is a compact operator from $L^{2}(0, \pi)$ into $L^{2}(0, \pi)$. Since $P_{n} g \rightarrow g$ for all $g \in L^{2}(0, \pi)$, it is straightforward that on $L^{2}(0, \pi)$,

$$
\left\|\left(I-P_{n}\right) A_{e}^{-1} B_{e}\right\| \rightarrow 0 \text { as } n \rightarrow \infty,
$$

using the operator norm for bounded operators from $L^{2}(0, \pi)$ to $L^{2}(0, \pi)$. Combined with the unique solvability of (1.6) (or (1.1)), we can obtain a convergence theory for (3.6).

It follows by standard arguments (e.g., see Atkinson [2, p. 51]) that $\left(I+P_{n} A_{e}^{-1} B_{e}\right)^{-1}$ exists and is uniformly bounded for all sufficiently large $n$, say $n \geq N$ :

$$
\left\|\left(I+P_{n} A_{e}^{-1} B_{e}\right)^{-1}\right\| \leq M<\infty, \quad n \geq N .
$$

For convergence, use the identity

$$
\rho-\rho_{n}=\left(I+P_{n} A_{e}^{-1} B_{e}\right)^{-1}\left(I-P_{n}\right) \rho .
$$

Thus, if $\rho \in L^{2}(0, \pi)$, we have convergence of $\rho_{n}$ to $\rho$. If $\rho$ is a smooth function, then $\left(I-P_{n}\right) \rho$ converges rapidly to zero, and the same is true of the convergence of $\rho_{n}$ to $\rho$.

We could continue this analysis to obtain results on uniform convergence and on convergence in $H_{e}^{t}$ for $t>0$. (For example, the analysis given in McLean [10, Chapter 5] can be generalized to the present situation.) Instead, we will look more carefully at a discrete Galerkin variant of (3.5). To motivate it, we look at the linear system arising in the solution of (3.3).

Using the basis $\left\{\varphi_{j}\right\}$ of $(3.1)$, write $\rho_{n}(s)=\sum_{j=0}^{n} \alpha_{j} \varphi_{j}(s)$. Then from (3.3), $\left\{\alpha_{j}\right\}$ is determined by requiring

$$
\left(\left(A_{e}+B_{e}\right) \rho_{n}, \varphi_{i}\right)=\left(f, \varphi_{i}\right), \quad i=0, \ldots, n,
$$

with the inner product $(f, g)=\int_{0}^{\pi} f(s) g(s) d s$. Noting that

$$
A_{e} \varphi_{j}(s)=\frac{1}{\max \{1, j\}} \varphi_{j}(s), \quad j \geq 0,
$$

we obtain the linear system

$$
\begin{gathered}
\pi \alpha_{0}+\sum_{j=0}^{n} \alpha_{j}\left(B_{e} \varphi_{j}, \varphi_{0}\right)=\left(f, \varphi_{0}\right), \\
\frac{\pi \alpha_{i}}{2 i}+\sum_{j=0}^{n} \alpha_{j}\left(B_{e} \varphi_{j}, \varphi_{i}\right)=\left(f, \varphi_{i}\right), \quad i=1, \ldots, n .
\end{gathered}
$$

The matrix elements are double integrals, and the right sides are single integrals. These integrals will be approximated to obtain a discrete Galerkin method.

\section{The DisCrete Galerkin Method}

To define the needed numerical integration in the approximation of (3.9), introduce the nodes 


$$
t_{j}=j h \text { for all integers } j, h=2 \pi /(2 n+1) .
$$

Let $C_{p, e}(2 \pi)$ denote the continuous functions that are even and periodic with period $2 \pi$. For $f \in C_{p, e}(2 \pi)$, approximate

$$
I(f)=\int_{0}^{\pi} f(t) d t
$$

by

$$
I_{n}(f)=h \sum_{j=0}^{n} f\left(t_{j}\right) \equiv \frac{h}{2} f(0)+h \sum_{j=1}^{n} f\left(t_{j}\right) .
$$

This somewhat unusual rule is derived from the $(2 n+1)$-point trapezoidal rule on $[0,2 \pi]$, taking into account that $f$ is even and periodic:

$$
\begin{aligned}
\int_{0}^{\pi} f(t) d t & =\frac{1}{2} \int_{-\pi}^{\pi} f(t) d t=\frac{1}{2} \int_{0}^{2 \pi} f(t) d t \\
& \doteq \frac{h}{2} \sum_{j=0}^{2 n+1} f\left(t_{j}\right) \equiv \frac{1}{2} T_{2 n+1}(f),
\end{aligned}
$$

where $T_{2 n+1}$ is the $(2 n+1)$-point trapezoidal rule (the notation " means to halve the first and last terms before summing); and from the evenness and periodicity of $f$, the right side of (4.3) is simply the definition $I_{n}(f)$ of $(4.2)$.

The choice in (4.1) of an odd number of points $t_{j}$ within each period $2 \pi$ results in the quadrature rule (4.2) having different behavior at the two ends of the integration interval $[0, \pi]$ : the left-hand end point is a quadrature point, the right-hand end point is not. This unsymmetrical choice, while perhaps not the preferred option from a practical point of view, is dictated by our desire to use certain theoretical results on interpolation with respect to trigonometric polynomials, which seem to be available only for the case of an odd number of interpolation points within each period. Applying the trapezoidal rule on $[-\pi, \pi]$ in the above derivation, we obtain a similar unsymmetric formula, with $\pi$ now a node point, but zero not one. Empirical results with the standard midpoint and trapezoidal rules replacing (4.2) give virtually the same numerical results, which would be expected from results on numerical integration and the essential equivalence of the midpoint and trapezoidal rules.

The following result is straightforward to prove. It shows that $I_{n}(f)$ preserves the orthogonality and normalization of the basis $\left\{\varphi_{0}, \ldots, \varphi_{n}\right\}$.

Lemma 1. We have

$$
I_{n}\left(\varphi_{j} \varphi_{k}\right)= \begin{cases}0, & j \neq k, 0 \leq j, k \leq n \\ \pi, & j=k=0 \\ \pi / 2, & 1 \leq j=k \leq n\end{cases}
$$


To approximate the integral operator $B_{e}$, use

$$
\left.B_{e, n} \rho(t)=I_{n}\left(b_{e}(t, \cdot)\right)(\cdot)\right)=h \sum_{j=0}^{n} b_{e}\left(t, t_{j}\right) \rho\left(t_{j}\right) .
$$

To approximate the inner products in (3.9), introduce the discrete (semidefinite) inner product

$$
(f, g)_{h}=I_{n}(f g)=h \sum_{j=0}^{n} f\left(t_{j}\right) g\left(t_{j}\right) .
$$

We could now approximate all integrals in the system (3.9), replacing $B_{e}$ with $B_{e, n}$, and replacing $(\cdot, \cdot)$ with $(\cdot, \cdot)_{h}$. Instead, we use a different but equivalent approach, better suited to showing convergence of the resulting method.

Introduce the seminorm

$$
|f|_{h}=\sqrt{(f, f)_{h}} .
$$

Then Lemma 1 yields

$$
|f|_{h}=\|f\|_{0}, \quad f \in \mathscr{X}_{n} .
$$

As in Atkinson and Bogomolny [4], introduce a discrete orthogonal projection operator $Q_{n}: C_{p, e}(2 \pi) \rightarrow \mathscr{X}_{n}$ by

$$
\left(Q_{n} f, \varphi\right)_{h}=(f, \varphi)_{h} \text { for all } \varphi \in \mathscr{X}_{n} .
$$

This definition can be extended to integration schemes with more than $n+1$ nodes, but the present definition is sufficient for our purposes. See Atkinson and Bogomolny [4] for a more general framework. (For an analysis of the discrete Galerkin method when more than $n+1$ integration nodes are being used, the results in McLean [10, Chapter 5, $\S 4$ ] can be generalized to the present situation.)

Lemma 2. The operator $Q_{n}$ satisfies the following properties as an operator on $C_{p, e}(2 \pi)$ :

(1) $Q_{n}^{2}=Q_{n}$;

(2) $\left(Q_{n} f, g\right)_{h}=\left(f, Q_{n} g\right)_{h}, f, g \in C_{p, e}(2 \pi)$;

(3) $Q_{n} f\left(t_{j}\right)=f\left(t_{j}\right), j=0, \ldots, n, f \in C_{p, e}(2 \pi)$.

The proofs of these results are straightforward and we omit them; see Atkinson and Bogomolny [4]. The last property says that $Q_{n} f$ is the trigonometric polynomial in $\mathscr{X}_{n}$ that interpolates $f$ at $t_{0}, \ldots, t_{n}$. Thus, $Q_{n}$ is both an approximation to the orthogonal projection $P_{n}$ and is the interpolating projection operator. Explicitly,

$$
Q_{n} f(s)=\frac{2}{\pi} \sum_{j=0}^{n}\left(f, \varphi_{j}\right)_{h} \varphi_{j}(s),
$$

with $\varphi_{j}(s)=\cos (j s)$. 
In Atkinson [3], a discrete orthogonal projection operator $\widetilde{Q}_{n}$, projecting onto the set of all trigonometric polynomials of degree $\leq n$ on $2 \pi$, was defined similarly. Let $C_{p}(2 \pi)$ denote the continuous periodic functions of period $2 \pi$, and let $\widetilde{\mathscr{X}}_{n}$ denote the trigonometric polynomials of degree $\leq n$. With the inner product based on the trapezoidal rule on $[0,2 \pi]$, as indicated in (4.3), $\widetilde{Q}_{n}$ was defined by the analogue of (4.8). Thus, the present operator is given by the restriction of $\tilde{Q}_{n}$ to the subspace $C_{p, e}(2 \pi)$ :

$$
Q_{n}=\left.\widetilde{Q}_{n}\right|_{C_{p, e}(2 \pi)} .
$$

This means the results about $\widetilde{Q}_{n}$ in the earlier paper can be used in this paper in discussing $Q_{n}$. For an explicit formula for $\widetilde{Q}_{n}$,

$$
\begin{array}{rlrl}
\widetilde{Q}_{n} f(s) & =\frac{1}{2 \pi} \sum_{j=-n}^{n} \alpha_{j} e^{i j s}, & f \in C_{p}(2 \pi), \\
\alpha_{j} & =h \sum_{k=0}^{2 n} f\left(t_{k}\right) e^{-i j t_{k}}, & t_{k} & =k h .
\end{array}
$$

For a more extensive discussion of $\widetilde{Q}_{n}$, see Atkinson [3]. The result (4.10) can be obtained from this formula for $\widetilde{Q}_{n}$ by assuming $f \in C_{p, e}(2 \pi)$ and then simplifying and rearranging the formula.

The approximate scheme. We approximate the Galerkin method (3.5) by

$$
\left(A_{e}+Q_{n} B_{e, n}\right) \psi_{n}=Q_{n} f, \quad \psi_{n} \in C_{p, e}(2 \pi),
$$

thus replacing $P_{n}$ and $B_{e}$ by $Q_{n}$ and $B_{e, n}$, respectively. Note that if the equation (4.11) is solvable, then

$$
A_{e} \psi_{n}=Q_{n}\left(f-B_{e, n} \psi_{n}\right) \in \mathscr{X}_{n} .
$$

The definition of $A_{e}$ then implies $\psi_{n} \in \mathscr{X}_{n}$. Thus, (4.11) can be written in the equivalent form

$$
Q_{n}\left(A_{e}+B_{e, n}\right) \psi_{n}=Q_{n} f, \quad \psi_{n} \in \mathscr{X}_{n} .
$$

To obtain a linear system for finding $\psi_{n}$, let

$$
\psi_{n}(s)=\sum_{j=0}^{n} \beta_{j} \varphi_{j}(s) .
$$

Calculate the coefficients $\left\{\beta_{j}\right\}$ by substituting (4.13) into (4.12) and then using (4.8) and (3.8):

$$
\begin{aligned}
& \pi \beta_{0}+\sum_{j=0}^{n} \beta_{j}\left(B_{e, n} \varphi_{j}, \varphi_{0}\right)_{h}=\left(f, \varphi_{0}\right)_{h}, \\
& \frac{\pi \beta_{i}}{2 i}+\sum_{j=0}^{n} \beta_{j}\left(B_{e, n} \varphi_{j}, \varphi_{i}\right)_{h}=\left(f, \varphi_{i}\right)_{h}, \quad i=1, \ldots, n .
\end{aligned}
$$


This is the discretization of the Galerkin system (3.9) that was suggested following (4.5). However, we now have the tools needed to analyze its convergence.

An alternative to (4.14) is obtained by using in (4.12) the interpolation property of $Q_{n}$, given in Lemma 2 (property (3)):

$$
\sum_{j=0}^{n} \beta_{j}\left\{\frac{\varphi_{j}\left(t_{i}\right)}{\max \{1, j\}}+B_{e, n} \varphi_{j}\left(t_{i}\right)\right\}=f\left(t_{i}\right), \quad i=0, \ldots, n .
$$

This system is faster to set up than (4.14), and it is essentially equivalent in conditioning to the system (4.14).

To be more precise about the conditioning of systems (4.14) and (4.15), we introduce some needed auxiliary matrices. Define

$$
\begin{gathered}
\Phi=\left[\Phi_{i j}\right]_{i, j=0}^{n}, \quad \Phi_{i j}=\varphi_{j}\left(t_{i}\right), \quad \Psi=D_{1}^{1 / 2} \Phi D_{2}^{-1 / 2}, \\
D_{1}=\operatorname{Diag}[h / 2, h, h, \ldots, h], \quad D_{2}=\operatorname{Diag}[\pi, \pi / 2, \ldots, \pi / 2] .
\end{gathered}
$$

These are all square matrices of order $n+1$. It can be shown that

$$
\Phi^{\mathrm{T}} D_{1} \Phi=D_{2}
$$

and, from this, that $\Psi$ is orthogonal, $\Psi^{\mathrm{T}} \Psi=I$.

Denote the systems (4.14) and (4.15) by

$$
M_{G} \boldsymbol{\beta}=\mathbf{f}_{G}, \quad M_{C} \boldsymbol{\beta}=\mathbf{f}_{C},
$$

respectively. Then

$$
M_{G}=\Phi^{\mathrm{T}} D_{1} M_{C}, \quad \mathbf{f}_{G}=\Phi^{\mathrm{T}} D_{1} \mathbf{f}_{C} .
$$

Manipulating this, we obtain

$$
\widehat{M}_{G}=\Psi^{\mathrm{T}} \widehat{M}_{C}
$$

with

$$
\widehat{M}_{G}=D_{2}^{1 / 2} M_{G}, \quad \widehat{M}_{C}=D_{1}^{1 / 2} M_{C} .
$$

The relation (4.18) says $\widehat{M}_{G}$ and $\widehat{M}_{C}$ have equal condition numbers, when using the operator norm based on the Euclidean vector norm. The matrices $\widehat{M}_{G}$ and $\widehat{M}_{C}$ are obtained from $M_{G}$ and $M_{C}$, respectively, by a simple (almost constant) rescaling in the rows, with the first row having a different weight than the remaining rows. This justifies our original statement that (4.14) and (4.15) are essentially equal in their conditioning.

\section{Convergence analysis}

Rewrite the integral equation (1.6) as

$$
\left(I+A_{e}^{-1} B_{e}\right) \rho=A_{e}^{-1} f,
$$

and rewrite the discrete Galerkin equation (4.11) as

$$
\left(I+A_{e}^{-1} Q_{n} B_{e, n}\right) \psi_{n}=A_{e}^{-1} Q_{n} f .
$$


The convergence analysis is carried out in the Banach space $C_{p, e}(2 \pi)$ equipped with the uniform norm $\|\cdot\|_{\infty}$. Note that $A_{e}^{-1} B_{e}$ is a compact operator on $C_{p, e}(2 \pi)$. The analysis of the solvability and convergence of (5.2) is carried out by using the framework of collectively compact operator approximations (see Anselone [1]). As notation, the letter $c$ is used as a generic constant in the following proofs.

Theorem 3. (1) Assume the curve $S$ has a $C^{\infty}$ parametrization, as in (1.2). Then the family $\left\{A_{e}^{-1} Q_{n} B_{e, n} \mid n \geq 1\right\}$ is collectively compact and converges pointwise to $A_{e}^{-1} B_{e}$ on $C_{p, e}(2 \pi)$.

(2) Assume the integral equation (1.6) is uniquely solvable for all $f \in H_{e}^{1}$ (or, equivalently, assume $C_{S} \neq 1$ ). Then for all sufficiently large $n$, say $n \geq N$, the operators $I+A_{e}^{-1} Q_{n} B_{e, n}$ are invertible on $C_{p, e}(2 \pi)$ and satisfy

$$
\left\|\left(I+A_{e}^{-1} Q_{n} B_{e, n}\right)^{-1}\right\| \leq M<\infty, \quad n \geq N .
$$

(3) For the error in the discrete Galerkin solution $\psi_{n}$ of (5.2),

$$
\left\|\rho-\psi_{n}\right\|_{\infty} \leq M\left\{\left\|A_{e}^{-1}\left(f-Q_{n} f\right)\right\|_{\infty}+\left\|A_{e}^{-1} B_{e} \rho-A_{e}^{-1} Q_{n} B_{e, n} \rho\right\|_{\infty}\right\} .
$$

Proof. A proof of part (1) can be given in two different ways. One way is to make use of (2.13) and (4.10), so as to regard $A_{e}^{-1} Q_{n} B_{e, n}$ as the restriction to $C_{p, e}(2 \pi)$ of a corresponding operator for the equation $(A+B) \rho=f$ on $C_{p}(2 \pi)$. Then (1) can be shown by invoking results from Atkinson [3] for the equation $(A+B) \rho=f$.

As an alternative, we can just imitate the proof of Atkinson [3]. We choose this approach so as to also introduce some notation needed later. We only indicate briefly the form of the proof, referring the reader to the earlier paper. Using the formula (4.4) for $B_{e, n}$, we write

$$
Q_{n} B_{e, n} f(t)=h \sum_{j=0}^{n} b_{e, n}\left(t, t_{j}\right) f\left(t_{j}\right)
$$

with $b_{e, n}(t, s)$ the cosine polynomial that interpolates $b_{e}(t, s)$ with respect to $t$ at $t_{0}, \ldots, t_{n}$, for all $s$ :

$$
b_{e, n}\left(t_{i}, s\right)=b_{e}\left(t_{i}, s\right), \quad i=0, \ldots, n, b_{e, n}(\cdot, s) \in \mathscr{X}_{n} .
$$

Define

$$
\left(A_{e}^{-1} Q_{n} B_{e, n}\right) f(t) \equiv C_{e, n} f(t)=h \sum_{j=0}^{n} c_{e, n}\left(t, t_{j}\right) f\left(t_{j}\right)
$$

The function $c_{e, n}(t, s) \equiv c_{e, n, s}(t)$ is defined by

$$
c_{e, n, s}=A_{e}^{-1} b_{e, n, s}, \quad b_{e, n, s}(t)=b_{e, n}(t, s) .
$$

Similarly, define

$$
\left(A_{e}^{-1} B_{e}\right) f(t) \equiv C_{e} f(t)=\int_{0}^{\pi} c_{e}(t, s) f(s) d s
$$


with $c_{e}(t, s)=c_{e, s}(t), c_{e, s}=A_{e}^{-1} b_{e, s}, b_{e, s}(t)=b_{e}(t, s)$. It can be shown that

$$
\sup _{t, s}\left|\frac{\partial^{l} c_{e}(t, s)}{\partial t^{l}}-\frac{\partial^{l} c_{e, n}(t, s)}{\partial t^{l}}\right| \rightarrow 0 \text { as } n \rightarrow \infty
$$

for all $l \geq 0$. Using this and (5.6), part (1) of the theorem will follow easily.

By the assumption of unique solvability for (1.6), $\left(I+C_{e}\right)^{-1}$ exists. Then by part (1) and Theorem 1.10 of Anselone [1], $\left(I+C_{e, n}\right)^{-1}$ exists and these inverses are uniformly bounded. For the convergence of $\psi_{n}$ to $\rho$, use the identity

$$
\left(I+C_{e, n}\right)\left(\rho-\psi_{n}\right)=A_{e}^{-1}\left(f-Q_{n} f\right)-\left(C_{e}-C_{e, n}\right) \rho .
$$

The convergence rates given in Atkinson [3] were based on error results for the derivatives of trigonometric interpolation polynomials. We give improved results here, using the following error results for trigonometric polynomial interpolation and for the trapezoidal numerical integration rule.

Lemma 4. Assume $f \in H^{t}, t>\frac{1}{2}$. Then for the trigonometric interpolation polynomial $\widetilde{Q}_{n} f$,

$$
\left\|f-\tilde{Q}_{n} f\right\|_{s} \leq \frac{c}{n^{t-s}}\|f\|_{t}, \quad t>s \geq 0 .
$$

(For the definition of $\widetilde{Q}_{n} f$, see the paragraph preceding $(4.10)$. It is the trigonometric polynomial of degree $\leq n$ that interpolates $f$ at the nodes $t_{j}=$ $2 \pi j /(2 n+1), j=0, \ldots, 2 n$.)

Proof. This result is taken from Chandler [5]. Its proof is based on using the Fourier series representation of $f$, given in (2.1), to obtain a Fourier series representation for $\widetilde{Q}_{n} f$. This is then subtracted from that for $f$, and the remaining terms are then bounded to give the result (5.9).

Lemma 5. Assume $f \in H^{t}$, with $t>\frac{1}{2}$. Let $I(f)$ denote the integral of $f$ over $[0,2 \pi]$, and let $T_{m}(f)$ denote the trapezoidal rule for approximating $I(f)$ :

$$
T_{m}(f)=h \sum_{j=0}^{m} f\left(t_{j}\right), \quad t_{j}=j h, h=\frac{2 \pi}{m} .
$$

Then the error in $T_{m}(f)$ satisfies

$$
\left|I(f)-T_{m}(f)\right| \leq \frac{\sqrt{4 \pi \zeta(2 t)}}{m^{t}}\|f\|_{t} .
$$

Proof. It is straightforward to prove that

$$
T_{m}\left(e^{i k s}\right)= \begin{cases}2 \pi, & k \equiv 0(\bmod m) \\ 0, & k \neq 0(\bmod m)\end{cases}
$$


Using this, apply $T_{m}(f)$ to the Fourier series representation of $f$ given in (2.1). Then

$$
I(f)-T_{m}(f)=-\sqrt{2 \pi} \sum_{\substack{k=-\infty \\ k \neq 0}}^{\infty} \hat{f}(k m)=-\sqrt{2 \pi} \sum_{\substack{k=-\infty \\ k \neq 0}}^{\infty} \hat{f}(k m)(k m)^{t}(k m)^{-t} .
$$

Using the Cauchy-Schwarz inequality,

$$
\begin{aligned}
\left|I(f)-T_{m}(f)\right| & \leq \sqrt{2 \pi}\left[\sum_{\substack{k=-\infty \\
k \neq 0}}^{\infty}|\hat{f}(k m)|^{2}|k m|^{2 t}\right]^{1 / 2}\left[\sum_{\substack{k=-\infty \\
k \neq 0}}^{\infty}|k m|^{-2 t}\right]^{1 / 2} \\
& \leq \sqrt{2 \pi}\|f\|_{t} m^{-t} \sqrt{2 \zeta(2 t)} .
\end{aligned}
$$

Theorem 6. Assume the hypotheses of Theorem 3. Also assume $f \in H_{e}^{r}$ with $r>1.5$. Then for all sufficiently large $n$,

$$
\left\|\rho-\psi_{n}\right\|_{\infty} \leq \frac{c(f, \varepsilon)}{n^{r-1.5-\varepsilon}}
$$

with $\varepsilon>0$ an arbitrary number sufficiently small that $r-1.5-\varepsilon>0$.

Proof. It follows from Yan and Sloan [12] that $\rho \in H_{e}^{r-1}$. Since $r-1>\frac{1}{2}$, the function $\rho$ is continuous, and thus $\rho \in C_{p, e}(2 \pi)$. To derive (5.12), we use Theorem 3(3).

To analyze $\left(C_{e}-C_{e, n}\right) \rho$, decompose it as

$$
\begin{aligned}
\left(C_{e}-C_{e, n}\right) \rho(t)= & {\left[\int_{0}^{\pi} c_{e}(t, s) \rho(s) d s-h \sum_{j=0}^{n}{ }^{\prime} c_{e}\left(t, t_{j}\right) \rho\left(t_{j}\right)\right] } \\
& +h \sum_{j=0}^{n}{ }^{\prime}\left[c_{e}\left(t, t_{j}\right)-c_{e, n}\left(t, t_{j}\right)\right] \rho\left(t_{j}\right) .
\end{aligned}
$$

For the final sum on the right side of (5.13), we need bounds on the speed of convergence of $c_{e, n}$ to $c_{e}$. Applying Lemma 4 to the convergence of $b_{e, n}$ and using the embedding of $C_{p, e}(2 \pi)$ in $H_{e}^{1}$ (or using results from Atkinson [3]), we have

$$
\max _{t, s}\left|b_{e}(t, s)-b_{e, n}(t, s)\right|=O\left(n^{-k}\right)
$$

for all $k>0$. Then from the definition of $c_{e}$ and $c_{e, n}$, the same is true of the convergence of $\left\{c_{e, n}\right\}$ to $c_{e}$ :

$$
\max _{t, s}\left|c_{e}(t, s)-c_{e, n}(t, s)\right|=O\left(n^{-k}\right)
$$

and this implies that the final sum on the right side of $(5.13)$ is $O\left(n^{-k}\right)$, uniformly in $t$, for all $k>0$.

Recall that the integration rule $I_{n}(f)$ of $(4.2)$ is simply the trapezoidal rule $T_{2 n+1}(f)$ applied to even periodic functions (see the derivation in (4.3)). Also 
recall from (5.6) that $c_{e}(t, s)$ is $C^{\infty}$ and periodic in both variables. Use Lemma 5 to show that the first term in brackets on the right side of (5.13),

$$
\int_{0}^{\pi} c_{e}(t, s) \rho(s) d s-h \sum_{j=0}^{n} c_{e}\left(t, t_{j}\right) \rho\left(t_{j}\right),
$$

is $O\left(n^{-r+1}\right)$, uniformly in $t$. Combining these results for (5.13), we have

$$
\left\|\left(C_{e}-C_{e, n}\right) \rho\right\|_{\infty}=O\left(n^{-r+1}\right) .
$$

To analyze $A_{e}^{-1}\left(f-Q_{n} f\right)$, use (2.9) to show that for any $t$ such that $r-1>$ $t>-\frac{1}{2}$,

$$
\begin{aligned}
\left\|A_{e}^{-1}\left(f-Q_{n} f\right)\right\|_{\infty} & \leq c\left\|A_{e}^{-1}\left(f-Q_{n} f\right)\right\|_{t} \\
& =c\left\|f-Q_{n} f\right\|_{t+1} \leq \frac{c}{n^{r-t-1}}\|f\|_{r},
\end{aligned}
$$

using Lemma 4 in the last step. To complete the proof, choose $t=\frac{1}{2}+\varepsilon$, with $\varepsilon$ sufficiently small. The constant $c$ depends on $\varepsilon$.

\section{APPLICATION TO POTENTIAL THEORY}

Consider solving the Dirichlet problem

$$
\begin{aligned}
& \Delta u(P)=0, \quad P \in \mathbb{R}^{2} \backslash S, \\
& u(P)=h(P), \quad P \in S, \\
& \sup _{P \in \mathbb{R}^{2}}|u(P)|<\infty .
\end{aligned}
$$

This problem has a unique solution.

We intend to use a single-layer potential to solve this problem; but by itself, a single-layer potential is not sufficient, because such a potential will generally be unbounded as $|P| \rightarrow \infty$. For this reason, we introduce the auxiliary equation

$$
\int_{S} \lambda(Q) \log |P-Q| d S(Q)=1, \quad P \in S .
$$

If the transfinite diameter $C_{S}$ of $S$ is not equal to 1 , then this equation has a unique solution $\lambda(P)$, differing only by a constant factor from the so-called equilibrium distribution. (See, for example, Sloan and Spence [11].) The transfinite diameter of $S$ is given by

$$
C_{S}=\exp \left[\left(\int_{S} \lambda(Q) d S(Q)\right)^{-1}\right] .
$$

Using $\lambda(Q)$, define the potential corresponding to $\lambda$ by

$$
v(P)=\int_{S} \lambda(Q) \log |P-Q| d S(Q), \quad P \in \mathbb{R}^{2} .
$$


To solve (6.1), first consider the single-layer potential

$$
w(P)=\int_{S} g(Q) \log |P-Q| d S(Q),
$$

where the distribution $g$ is obtained by solving

$$
\int_{S} g(Q) \log |P-Q| d S(Q)=h(P), \quad P \in S .
$$

As stated earlier in $\S 1$, this equation is uniquely solvable if $C_{S} \neq 1$. The potential $w(P)$ satisfies the boundary condition on $S$ given in (6.1), but in general is unbounded as $|P| \rightarrow \infty$ :

$$
w(P) \doteq(\log |P|) \int_{S} g(Q) d S(Q) \quad \text { as }|P| \rightarrow \infty .
$$

To obtain the desired solution of $(6.1)$, introduce

$$
\begin{aligned}
u(P) & =w(P)-\alpha v(P)+\alpha \\
& =\int_{S}[g(Q)-\alpha \lambda(Q)] \log |P-Q| d S(Q)+\alpha .
\end{aligned}
$$

This satisfies Laplace's equation and the Dirichlet condition of $(6.1)$ for any $\alpha$. We choose $\alpha$ so that the density function

$$
k=g-\alpha \lambda
$$

satisfies

$$
\int_{S} k(Q) d S(Q)=0
$$

This requires

$$
\alpha=\int_{S} g(Q) d S(Q) / \int_{S} \lambda(Q) d S(Q) .
$$

The condition (6.9) implies that the integral term in (6.7) will be bounded as $|P| \rightarrow \infty$. Thus, the resulting potential in (6.7) is a solution to the original problem (6.1).

In those cases where $C_{S}=1$ or is close to 1 , the problem (6.1) can easily be rescaled to another Dirichlet problem for Laplace's equation, with a smaller or larger curve $S$ whose transfinite diameter is not near 1 . For additional discussion on this means of solving (6.1), see Jaswon and Symm [9, pp. 54-56].

To construct the solution $u$ of $(6.1)$, we can use the discrete Galerkin method of this paper to solve both (6.2) and (6.6). Denote the approximate solutions by $\lambda_{n}$ and $g_{n}$, respectively. Substitute these into (6.10), numerically evaluating the integrals by the integration rule of (4.2). Denote the resulting approximation to $\alpha$ by $\alpha_{n}$. Using the convergence results of Theorem 6, the same order of convergence can be shown for $\alpha_{n} \rightarrow \alpha$.

Using this $\alpha_{n}$, define

$$
\begin{gathered}
k_{n}=g_{n}-\alpha_{n} \lambda_{n}, \\
u_{n}(P)=\int_{S} k_{n}(Q) \log |P-Q| d S(Q)+\alpha_{n} .
\end{gathered}
$$


One easily shows

$$
\begin{aligned}
u(P)-u_{n}(P) & =\int_{S}\left[k(Q)-k_{n}(Q)\right] \log |P-Q| d S(Q)+\alpha-\alpha_{n} \\
& =\int_{0}^{\pi}\left[\sigma(\tau)-\sigma_{n}(\tau)\right] \log |P-a(\tau)| d \tau+\alpha-\alpha_{n}
\end{aligned}
$$

where

$$
\begin{aligned}
\sigma(t) & =k(a(t))\left|r^{\prime}(\cos t)\right| \sin t, \\
\sigma_{n}(t) & =k_{n}(a(t))\left|r^{\prime}(\cos t)\right| \sin t .
\end{aligned}
$$

Thus,

$$
\left|u(P)-u_{n}(P)\right| \leq\left\|\sigma-\sigma_{n}\right\|_{\infty} \int_{0}^{\pi}|\log | P-a(\tau)|| d \tau+\left|\alpha-\alpha_{n}\right| .
$$

Again using Theorem 6, we now see that for fixed $P$, the rate of convergence of $u_{n}(P)$ to $u(P)$ is at least that in (5.12). From (6.13), the convergence rate is uniform on bounded regions of $\mathbb{R}^{2}$.

We further approximate $(6.12)$ by evaluating the integral numerically, using the integration rule of (4.2) with $q+1$ nodes. Denote the resulting approximation by $u_{n, q}(P)$. It can be shown that for each $P$, and for all sufficiently large $n$,

$$
\left|u(P)-u_{n, q}(P)\right| \leq \frac{d_{q}(P)}{n^{r-1.5-\varepsilon}}, \quad q \geq n .
$$

The constant $d_{q}(P)$ approaches $\infty$ as $P$ approaches $S$, because the integrand in (6.12) becomes singular in this case. For $P$ near $S$, it is better to use $u_{n, q}(P)$ with $q$ much larger than $n$. This is illustrated in the following section.

\section{NUMERICAL EXAMPLES}

We give three examples, to illustrate and further investigate the numerical methods studied in $\S \S 4$ through 6 . These examples can also be considered as illustrations of the solution of crack problems in the plane; for example, see Costabel et al. [6, §3]. All of the following calculations were performed in double-precision arithmetic on a microcomputer with an $80286 / 287$ processor.

(a) Consider solving the Dirichlet problem (6.1) for the true known potential

$$
u(x, y)=\operatorname{Real}\left[\exp \left(\sqrt{z^{2}-1}-z\right)\right], \quad z=x+i y .
$$

The branch cut for $\sqrt{z^{2}-1}$ is to be the interval $[-1,1]$, and the boundary curve $S$ is this same interval. The Dirichlet data $h$ in $(6.1)$ is generated from (7.1).

The transformation of the equation (1.1) into (1.4) yields the integral equation

$$
-\frac{1}{\pi} \int_{0}^{\pi} \rho(\tau) \log |\cos t-\cos \tau| d \tau=f(t), \quad 0 \leq t \leq \pi,
$$


with $f(t)=-\frac{1}{\pi} h(\cos t)$ and $\rho(t)=g(\cos t) \sin t$. Equivalently,

$$
g(x)=\frac{\rho\left(\cos ^{-1} x\right)}{\sqrt{1-x^{2}}}, \quad-1 \leq x \leq 1 .
$$

As $x \rightarrow \pm 1$, we obtain easily the asymptotic behavior of $g(x)$ :

$$
\begin{aligned}
& g(x) \doteq \frac{\rho(0) / \sqrt{2}}{\sqrt{1-x}} \quad \text { as } x \rightarrow 1, \\
& g(x) \doteq \frac{\rho(\pi) / \sqrt{2}}{\sqrt{1+x}} \quad \text { as } x \rightarrow-1 .
\end{aligned}
$$

Rather than giving values for $\rho(0)$ and $\rho(\pi)$, we give approximate values for $\sigma(0)$ and $\sigma(\pi)$, with

$$
\sigma(t)=k(\cos t) \sin t
$$

and $k=g-\lambda \sigma$, from (6.8). The function $k(Q) \equiv k(x),-1 \leq x \leq 1$, is more appropriate than $g(x)$ in light of the constructions of $\S 6$. The values of $\sigma_{n}(0)$ and $\sigma_{n}(\pi)$, together with their differences for successive $n$, are given in Table 1. The convergence is very rapid, as can be seen from the differences.

In Table 2, we give values of $u_{n, n}(x, y)$ for the four points

$$
\left(x_{i}, y_{i}\right)=(1.1,0),(.5,1),(.5, .01),(0,100)
$$

for $i=1,2,3,4$. The convergence is rapid at all points except the third, $(.5, .01)$. This point is very close to the curve $S=[-1,1]$, and consequently the integrand in (6.12) is very peaked. With a more accurate numerical integration, better values are obtained. For example, with $q=640$, the errors in $u_{n, q}$ act in a more predictable and well-behaved fashion; see Table 3.

(b) Again use $S=[-1,1]$; but use the Dirichlet data

$$
h(x)=(1+x) \log (1+x), \quad-1 \leq x \leq 1 .
$$

TABLE 1

Density values for (7.1)

$\begin{array}{rcrcc}n & \sigma_{n}(0) & \sigma_{n}(0)-\sigma_{n-1}(0) & \sigma_{n}(\pi) & \sigma_{n}(\pi)-\sigma_{n-1}(\pi) \\ & & & & \\ 2 & .09250969 & -5.61 \mathrm{E}-2 & -.51670183 & -3.68 \mathrm{E}-1 \\ 3 & .12422289 & 3.17 \mathrm{E}-2 & -.75022883 & -2.53 \mathrm{E}-1 \\ 4 & .11550568 & -8.72 \mathrm{E}-3 & -.83675543 & -8.65 \mathrm{E}-2 \\ 5 & .11738633 & 1.88 \mathrm{E}-3 & -.85960064 & -2.28 \mathrm{E}-2 \\ 6 & .11705635 & -3.30 \mathrm{E}-4 & -.86431966 & -4.72 \mathrm{E}-3 \\ 7 & .11710531 & 4.90 \mathrm{E}-5 & -.86512296 & -8.03 \mathrm{E}-4 \\ 8 & .11709901 & -6.30 \mathrm{E}-6 & -.86523943 & -1.16 \mathrm{E}-4 \\ 9 & .11709972 & 7.16 \mathrm{E}-7 & -.86525415 & -1.47 \mathrm{E}-5 \\ 10 & .11709966 & -7.30 \mathrm{E}-8 & -.86525580 & -1.65 \mathrm{E}-6\end{array}$


TABLE 2

\begin{tabular}{rcrrr}
\multicolumn{5}{c}{ Errors in $u_{n, n}\left(x_{i}, y_{i}\right)$} \\
$n$ & $i=1$ & \multicolumn{1}{c}{$i=2$} & $i=3$ & $i=4$ \\
& & & & \\
2 & $3.28 \mathrm{E}-4$ & $4.96 \mathrm{E}-3$ & $1.06 \mathrm{E}-1$ & $8.33 \mathrm{E}-3$ \\
3 & $6.25 \mathrm{E}-3$ & $1.14 \mathrm{E}-4$ & $-2.38 \mathrm{E}-2$ & $1.98 \mathrm{E}-4$ \\
4 & $1.03 \mathrm{E}-3$ & $-1.21 \mathrm{E}-5$ & $-8.42 \mathrm{E}-2$ & $2.75 \mathrm{E}-6$ \\
5 & $4.66 \mathrm{E}-4$ & $-9.81 \mathrm{E}-7$ & $1.39 \mathrm{E}-2$ & $2.50 \mathrm{E}-8$ \\
6 & $1.52 \mathrm{E}-4$ & $-3.56 \mathrm{E}-8$ & $7.67 \mathrm{E}-5$ & $1.60 \mathrm{E}-10$ \\
7 & $5.55 \mathrm{E}-5$ & $5.91 \mathrm{E}-8$ & $4.83 \mathrm{E}-2$ & $7.61 \mathrm{E}-13$ \\
8 & $2.02 \mathrm{E}-5$ & $-1.16 \mathrm{E}-8$ & $8.74 \mathrm{E}-3$ & $1.78 \mathrm{E}-15$ \\
9 & $7.46 \mathrm{E}-6$ & $1.21 \mathrm{E}-9$ & $2.76 \mathrm{E}-3$ & $-1.44 \mathrm{E}-15$ \\
10 & $2.79 \mathrm{E}-6$ & $-2.79 \mathrm{E}-11$ & $-3.28 \mathrm{E}-2$ & $-1.11 \mathrm{E}-15$
\end{tabular}

TABLE 3

Errors in $u_{n, q}(.5, .01)$ with $q=640$

\begin{tabular}{rrrr}
$n$ & \multicolumn{1}{c}{ Error } & $n$ & \multicolumn{1}{l}{ Error } \\
& & & \\
1 & $-2.04 \mathrm{E}-1$ & 6 & $7.19 \mathrm{E}-5$ \\
2 & $4.39 \mathrm{E}-2$ & 7 & $-1.80 \mathrm{E}-5$ \\
3 & $1.30 \mathrm{E}-2$ & 8 & $9.90 \mathrm{E}-7$ \\
4 & $-5.48 \mathrm{E}-3$ & 9 & $1.04 \mathrm{E}-7$ \\
5 & $4.68 \mathrm{E}-4$ & 10 & $-1.90 \mathrm{E}-8$
\end{tabular}

This function is not continuously differentiable, although it does belong to $H^{1}(-1,1)$. The change of variable $x=\cos t$ yields

$$
f(t)=-\frac{1}{\pi}(1+\cos t) \log (1+\cos t), \quad 0 \leq t \leq \pi .
$$

This function is once, but not twice, continuously differentiable. However $f \in$ $H_{e}^{2}$, and in fact, it can be shown that $f \in H_{e}^{2.5-\varepsilon}$ for any $\varepsilon>0$.

The function $f$ satisfies the smoothness assumption of Theorem 6 , which predicts an $O\left(n^{-1+2 \varepsilon}\right)$ rate of convergence. The empirical results agree with this rate. In Table 4, we give values of $\sigma_{n}(0)$ and $\sigma_{n}(\pi)$, together with their differences for various values of $n$. In the table, $D_{n}=\sigma_{n}-\sigma_{n / 2}$ and Ratio denotes the ratio of successive values of $D_{n}$. Empirically as $n$ increases,

$$
\sigma(\pi)-\sigma_{n}(\pi) \doteq \frac{c}{n} \quad \text { or } \quad \frac{c}{n} \log n
$$

for some constant $c$. This is the worst behavior in $\sigma_{n}(t)$, as might be expected because of the singularity at $x=-1$. It conforms rather closely with the rate 
TABLE 4

Density values $\sigma_{n}$ for (7.4)

\begin{tabular}{rccrccr}
$n$ & $\sigma_{n}(0)$ & \multicolumn{1}{c}{$D_{n}(0)$} & Ratio & $\sigma_{n}(\pi)$ & $D_{n}(\pi)$ & Ratio \\
2 & -.427405 & $-5.97 \mathrm{E}-2$ & & .111365 & $-2.56 \mathrm{E}-1$ & \\
4 & -.417405 & $1.00 \mathrm{E}-2$ & -6.0 & -.040274 & $-1.52 \mathrm{E}-1$ & 1.69 \\
8 & -.416130 & $1.28 \mathrm{E}-3$ & 7.8 & -.125819 & $-8.55 \mathrm{E}-2$ & 1.77 \\
16 & -.415998 & $1.31 \mathrm{E}-4$ & 9.7 & -.171888 & $-4.61 \mathrm{E}-2$ & 1.86 \\
32 & -.415986 & $1.28 \mathrm{E}-5$ & 10.2 & -.195900 & $-2.40 \mathrm{E}-2$ & 1.92
\end{tabular}

TABLE 5

Errors in $u_{n, n}\left(x_{i}, y_{i}\right)$

\begin{tabular}{rrccr}
$n$ & $i=1$ & $i=2$ & $i=3$ & \multicolumn{1}{c}{$i=4$} \\
1 & $2.84 \mathrm{E}-1$ & $6.38 \mathrm{E}-1$ & $-6.79 \mathrm{E}-1$ & $-2.48 \mathrm{E}-1$ \\
2 & $-5.71 \mathrm{E}-2$ & $2.13 \mathrm{E}-1$ & $1.50 \mathrm{E}-3$ & $1.02 \mathrm{E}-2$ \\
3 & $-4.07 \mathrm{E}-2$ & $1.46 \mathrm{E}-1$ & $-1.75 \mathrm{E}-1$ & $3.93 \mathrm{E}-3$ \\
4 & $-1.52 \mathrm{E}-2$ & $1.15 \mathrm{E}-1$ & $-2.55 \mathrm{E}-2$ & $-9.82 \mathrm{E}-4$ \\
5 & $-3.23 \mathrm{E}-3$ & $9.30 \mathrm{E}-2$ & $-7.69 \mathrm{E}-2$ & $7.52 \mathrm{E}-5$ \\
6 & $1.74 \mathrm{E}-4$ & $7.49 \mathrm{E}-2$ & $-2.45 \mathrm{E}-2$ & $3.78 \mathrm{E}-6$ \\
7 & $7.15 \mathrm{E}-4$ & $6.05 \mathrm{E}-2$ & $-4.89 \mathrm{E}-2$ & $-1.07 \mathrm{E}-6$ \\
8 & $5.05 \mathrm{E}-4$ & $4.93 \mathrm{E}-2$ & $-2.06 \mathrm{E}-2$ & $1.90 \mathrm{E}-9$ \\
9 & $2.27 \mathrm{E}-4$ & $4.05 \mathrm{E}-2$ & $-3.53 \mathrm{E}-2$ & $1.19 \mathrm{E}-8$ \\
10 & $6.07 \mathrm{E}-5$ & $3.33 \mathrm{E}-2$ & $-1.72 \mathrm{E}-2$ & $4.00 \mathrm{E}-10$
\end{tabular}

of convergence given by Theorem 6 . The empirical rate of convergence of the approximating potentials $u_{n, q}(x, y)$ is much better than that of $\sigma_{n}$ to $\sigma$.

(c) Let $S$ be the upper half of the ellipse

$$
x^{2}+4 y^{2}=1
$$

with $y \geq 0$. For a parametrization, use

$$
r(\theta)=\left(\sin \left(\frac{\pi}{2} \theta\right), .5 \cos \left(\frac{\pi}{2} \theta\right)\right), \quad-1 \leq \theta \leq 1 .
$$

For the boundary data, we take

$$
h(x, y)=e^{x}, \quad(x, y) \in S .
$$

The true solution is unknown in this case; the errors given below are based on answers $u_{n, q}$ computed with much larger $n$ and $q$.

In Table 5, we give empirical results for the error in $u_{n, n}(x, y)$ at selected points $(x, y)$. In particular, denote

$$
\left(x_{i}, y_{i}\right)=(1.1,0),(1.001,0),(0, .49),(0,10)
$$




\begin{tabular}{rcccc}
\multicolumn{5}{c}{ TABLE 6} \\
\multicolumn{5}{c}{ Errors in $u_{8, q}\left(x_{i}, y_{i}\right)$} \\
$q$ & $i=1$ & $i=2$ & $i=3$ & $i=4$ \\
& & & & \\
8 & $5.05 \mathrm{E}-4$ & $4.93 \mathrm{E}-2$ & $-2.06 \mathrm{E}-2$ & $1.90 \mathrm{E}-9$ \\
20 & $3.17 \mathrm{E}-5$ & $2.17 \mathrm{E}-3$ & $-8.31 \mathrm{E}-3$ & $8.80 \mathrm{E}-9$ \\
40 & $3.17 \mathrm{E}-5$ & $-1.94 \mathrm{E}-3$ & $-2.97 \mathrm{E}-3$ & $8.80 \mathrm{E}-9$ \\
80 & $3.17 \mathrm{E}-5$ & $-3.78 \mathrm{E}-4$ & $-6.71 \mathrm{E}-4$ & $8.80 \mathrm{E}-9$ \\
160 & $3.17 \mathrm{E}-5$ & $-3.78 \mathrm{E}-4$ & $-6.71 \mathrm{E}-4$ & $8.80 \mathrm{E}-9$
\end{tabular}

for $i=1,2,3,4$. The arc $S$ has the endpoints $( \pm 1,0)$. In solving the equation (1.4), the singular behavior of the solution $g(Q)$ of $(1.1)$ is easily recovered from the solution $\rho(t)$ and the transformation in (1.5), just as was done in (7.3).

The error in $u_{n, n}(x, y)$ is relatively large at $(1.001,0)$ and $(0, .49)$, both of which are very close to the curve $S$. To show the improvement in the error that is possible with a larger number $q$ of quadrature nodes used in evaluating $u_{n}(x, y)$, we give results for $u_{8, q}(x, y)$ with varying $q$. These results are given in Table 6. The needed value of $q$ varies with the point $(x, y)$, with the value increasing as $(x, y)$ approaches $S$.

\section{ACKNOWLEDGMENTS}

The work of the first author was carried out while he was a Visiting Professor in the School of Mathematics of the University of New South Wales; he was supported in part by the University of New South Wales ARC program grant "Numerical analysis for integrals, integral equations, and boundary value problems". Both authors would also like to thank Graeme Chandler, University of Queensland, and William McLean, University of Tasmania, for their assistance in finding the needed theory and literature on the approximation of periodic functions by trigonometric polynomials. In addition, the authors would like to thank the referees for their suggestions.

\section{BIBLIOGRAPHY}

1. P. Anselone, Collectively compact operator approximation theory, Prentice-Hall, Englewood Cliffs, N. J., 1971.

2. K. Atkinson, A survey of numerical methods for Fredholm integral equations of the second kind, SIAM, Philadelphia, Pa., 1976.

3. __ A discrete Galerkin method for first kind integral equations with a logarithmic kernel, J. Integral Equations Appl. 1 (1988), 343-363.

4. K. Atkinson and A. Bogomolny, The discrete Galerkin method for integral equations, Math. Comp. 48 (1987), 595-616. 
5. G. Chandler, Private communication, 1988.

6. M. Costabel, V. Ervin, and E. Stephan, On the convergence of collocation methods for Symm's integral equation on open curves, Math. Comp. 51 (1988), 167-179.

7. G. Gladwell and S. Coen, A Chebyshev approximation method for microstrip problems, IEEE Trans. Microwave Theory Tech. 23 (1975), 865-870.

8. G. Hsiao, E. Stephan, and W. Wendland, On the Dirichlet problem in elasticity for a domain exterior to an arc, Math. Institut A, Universität Stuttgart, Tech. Rep. 15, 1989.

9. M. Jaswon and G. Symm, Integral equation methods in potential theory and elastostatics, Academic Press, London, 1977.

10. W. McLean, Boundary integral methods for the Laplace equation, Ph.D. Thesis, Australian National University, Canberra, 1985.

11. I. H. Sloan and A. Spence, The Galerkin method for integral equations of the first kind with logarithmic kernel: Theory, IMA J. Numerical Anal. 8 (1988), 105-122.

12. Y. Yan and I. H. Sloan, On integral equations of the first kind with logarithmic kernels, J. Integral Equations Appl. 1 (1988), 549-579.

Department of Mathematics, University of Iowa, Iowa City, Iowa 52242

School of Mathematics, University of New South Wales, Sydney, New South Wales 2033, Australia 